\title{
A GENERALIZATION OF RAPP'S FORMULA
}

\author{
C. E. M. PEARCE
}

(Received 11 November 1980)

\begin{abstract}
The Rapp formula of teletraffic dimensioning is generalized to admit an arbitrary renewal stream of offered traffic. The derivation proceeds from a heavy traffic approximation and provides also an estimate of the order of error involved in the Rapp formula. In principle, the method could be used to seek convenient higher order approximations.

Our equations give an incidental theoretical substantiation of an empirical result relating to marginal occupancy found recently by Potter.
\end{abstract}

\section{Introduction}

Suppose a stream of calls has mean $M$ and variance $V$, that is, $M$ and $V$ are the mean and variance of the steady-state distribution of the number of occupied trunks induced by the stream in an infinite full-availability trunk group. The standard dimensioning problem in teletraffic utilizing the Wilkinson Equivalent Random Method [6] seeks to make the subsequent routing of the stream amenable to mathematical analysis by representing it as the overflow traffic resulting from a Poisson traffic of mean $A$ being offered to a full-availability group of $N$ trunks possessing negative exponential holding times. The formulae giving the values $M_{N}$ and $V_{N}$ resulting from offering a Poisson traffic of mean $A$ to $N$ negative exponential trunks do not lend themselves to exact analytical inversion and practical calculation usually proceeds via the approximate formulae

$$
A \simeq V+3 Z(Z-1)
$$

Copyright Australian Mathematical Society 1982 
and

$$
N=A(M+Z) /(M+Z-1)-M-1,
$$

where $Z=V / M$.

Formula (1), to which Rapp's name is attached, was found by him from numerical calculations. Relation (2) is exact if $A$ assumes its exact value (see [4]). When $V$ is less than $M$, the Wilkinson Equivalent Random Method produces a negative value for the number $N$ of trunks. Recently Potter [3] has proposed the use of an Equivalent Non-random Method in which a general renewal stream of calls is offered to a primary trunk group. This method may be implemented graphically in a fashion analogous to the traditional use of the Equivalent Random Method. The model is particularly appropriate in situations in which it is known that the observed traffic results from the overflow from the trunk group of some non-Poisson stream. The exact formulae expressing $V_{N}$ and $M_{N}$ in terms of the mean offered traffic $A$ and trunk group size $N$ (noted in the following section) in this general case are rather complicated and only numerical methods exist for the inversion problem.

Here we derive a simple and accurate generalization of Rapp's formula assuming heavy traffic, which usually obtains in practice. Our results subsume Rapp's formula for Poisson offered traffic, for which no analytical derivation appears to have been presented hitherto in the literature.

\section{The heavy traffic approximation}

Suppose inter-arrival times in the offered renewal stream have common distribution function $F(x)$ with

$$
\phi(x)=\int_{0}^{\infty} e^{-s x} d F(x), \quad \text { where Re. } s>0,
$$

and the holding times in the trunk group have mean $\mu^{-1}$. Since the equilibrium distribution of trunk occupancy is independent of time scale, we may regard $F$ and $\phi$ as fixed and represent variation in offered traffic intensity by changes in $\mu$. Conditions of heavy traffic then correspond to small $\mu$. In terms of $\phi, M_{N}, V_{N}$ and $A$ are prescribed by

$$
\begin{gathered}
A=-\left[\mu \phi^{\prime}(0)\right]^{-1}, \\
M_{N}=-\phi(\mu)\left[\mu \phi^{\prime}(0) \sum_{r=0}^{N-1}\left(\frac{N-1}{r}\right) k_{r}(\mu)\right]^{-1}
\end{gathered}
$$


and

$$
V_{N}=M_{N}\left[-M_{N}+\sum_{r=0}^{N}\left(\begin{array}{c}
N \\
r
\end{array}\right) k_{r}(\mu) /\left(\sum_{r=1}^{N}\left(\begin{array}{c}
N-1 \\
r-1
\end{array}\right) k_{r}(\mu)\right)\right]
$$

where

$$
k_{n}(s)=\sum_{j=1}^{n}[1-\phi(s+(j-1) \mu)] / \phi(s+j \mu), \quad n>1, \text { Re. } s>0,
$$

and $k_{0}(s)$ is taken as unity. These formulae result from combining results on the $G / M / N / N$ loss and $G / M / \infty$ systems due to Takács [5] and Cohen [1], and are given in the review paper [2].

From an elementary mean value theorem we have, for $j=1,2$ and 3, that

$$
\phi(j \mu)=1+\mu \phi_{1}+\left(j^{2} \mu^{2} / 2\right) \phi_{2 j},
$$

where $\phi_{1}=\phi^{\prime}(0)$ and $\phi_{2 j}=\phi^{\prime \prime}\left(\eta_{j}\right)$ for some $\eta_{j}$ where $0<\eta_{j}<j \mu$. Under conditions of heavy traffic when $\mu$ is small, substitution for $\phi$ in (6) yields

$$
\begin{gathered}
k_{1}(\mu)=-\mu \phi_{1}+\mu^{2}\left(2 \phi_{1}^{2}-\phi_{21} / 2\right)+\mu^{3} \phi_{1}\left(2 \phi_{22}+\phi_{21}-4 \phi_{1}^{2}\right) \\
+\mu^{4}\left(8 \phi_{1}^{4}-8 \phi_{1}^{2} \phi_{22}-2 \phi_{1}^{2} \phi_{21}+\phi_{21} \phi_{22}\right)+O\left(\mu^{5}\right), \\
k_{2}(\mu)=2 \mu^{2} \phi_{1}^{2}+\mu^{3} \phi_{1}\left(2 \phi_{22}+\phi_{21}-10 \phi_{1}^{2}\right) \\
+\mu^{4}\left(38 \phi_{1}^{4}-5 \phi_{1}^{2} \phi_{21}-14 \phi_{1}^{2} \phi_{22}-9 \phi_{1}^{2} \phi_{23}+\phi_{21} \phi_{22}\right)+O\left(\mu^{5}\right), \\
k_{3}(\mu)=-6 \mu^{3} \phi_{1}^{3}+\mu^{4} \phi_{1}^{2}\left(-3 \phi_{21}-6 \phi_{22}-9 \phi_{23}+54 \phi_{1}^{2}\right)+O\left(\mu^{5}\right), \\
k_{4}(\mu)=24 \mu^{4} \phi_{1}^{4}+O\left(\mu^{5}\right)
\end{gathered}
$$

and

$$
k_{n}(\mu)=O\left(\mu^{5}\right) \quad \text { for } n>4,
$$

provided $\phi^{\prime \prime}(0)$ exists.

These values may now be substituted in equations (4), (5) and (6) to provide

$$
\begin{gathered}
M_{N}=-\left(\mu \phi_{1}\right)^{-1}\left[1+N \mu \phi_{1}+\mu^{2}(N / 2) \phi_{21}\right. \\
\left.+\mu^{3} \phi_{1} N(N-1)\left(\phi_{21} / 2-\phi_{22}\right)+O\left(\mu^{4}\right)\right], \\
V_{N}=-\left(\mu \phi_{1}\right)^{-1}\left[\phi_{21} /\left(2 \phi_{1}^{2}\right)+\mu\left\{2 N \phi_{1}^{-1}\left(\phi_{21}-\phi_{22}\right)-\phi_{21}^{2} /\left(4 \phi_{1}^{3}\right)\right\}\right. \\
+\mu^{2}\left\{\phi_{21}^{3} /\left(8 \phi_{1}^{4}\right)+N \phi_{21} \phi_{22} / \phi_{1}^{2}-(N / 2) \phi_{21}^{2} / \phi_{1}^{2}+\phi_{21}\left(5 N^{2} / 2-N\right)\right. \\
\left.\left.-\phi_{22}\left(7 N^{2}-5 N\right)+\phi_{23}\left(9 N^{2}-9 N\right) / 2\right\}+O\left(\mu^{3}\right)\right]
\end{gathered}
$$

and

$$
\begin{aligned}
Z_{N}=\phi_{21} /\left(2 \phi_{1}^{2}\right)+\mu\left[(3 N / 2) \phi_{21} / \phi_{1}-2 N \phi_{22} / \phi_{1}-\phi_{21}^{2} /\left(4 \phi_{1}^{3}\right)\right] \\
+\mu^{2}\left[N(N-1)\left\{\phi_{21}-5 \phi_{22}+9 \phi_{23} / 2\right\}+N \phi_{21} \phi_{22} / \phi_{1}^{2}\right. \\
\left.-N \phi_{21}^{2} /\left(2 \phi_{1}^{2}\right)+\phi_{21}^{3} /\left(8 \phi_{1}^{4}\right)\right]+O\left(\mu^{3}\right)
\end{aligned}
$$




\section{Generalization of Rapp's formula}

Equations (8), (9) and (10) above hold for renewal offered traffic with $\phi^{\prime \prime}(0)<\infty$. We now suppose that $F$ has its first five moments finite, so that $\phi_{\imath}=\phi^{(i)}(0)$ exists for $1 \leqslant i \leqslant 5$. Then

$$
\phi_{2 j}=\phi_{2}+(j \mu / 3) \phi_{3}+\left(j^{2} \mu^{2} / 12\right) \phi_{4}+O\left(\mu^{3}\right), \quad \text { for } j=1,2,3,
$$

and we may deduce from (8), (9),.(10) and (3) that

$$
\begin{gathered}
M_{N}=A-N+A^{-1} N \alpha_{2}+O\left(A^{-2}\right), \\
V_{N}=A \alpha_{2}+\alpha_{2}^{2}-\alpha_{3}+A^{-1}\left[\alpha_{2}^{3}+\alpha_{4}-2 \alpha_{2} \alpha_{3}-N\left(\alpha_{2}+4 \alpha_{3}-2 \alpha_{2}^{2}\right)\right] \\
+O\left(A^{-2}\right)
\end{gathered}
$$

and

$$
Z_{N}=\alpha_{2}+A^{-1}\left(N \alpha_{2}+\alpha_{2}^{2}-\alpha^{3}\right)+O\left(A^{-2}\right),
$$

where $\alpha_{i}=\phi_{i} /\left(i ! \phi_{1}^{i}\right)$ for $i=2,3,4$.

The $\alpha_{i}$ are linear invariants of the distribution given by $F$, that is, they are characteristics of $\phi$ which are independent of the choice of time scale. It follows in particular that, under heavy traffic conditions, the peakedness factor $Z_{N}=$ $V_{N} / M_{N}$ is, to first order, an invariant of the overflow traffic, which goes some way to explaining the practical relevance of this quantity as opposed to the apparently more natural $V_{N} / M_{N}^{2}$.

Equations (12) and (13) may be combined to yield

$$
\begin{aligned}
& V_{N}+\alpha_{3}-\alpha_{2}^{2}+\left(\alpha_{2}^{-1}-2+4 \alpha_{3} / \alpha_{2}^{2}\right) Z_{N}\left(Z_{N}-\alpha_{2}\right) \\
& \quad=A \alpha_{2}+A^{-1}\left[\alpha_{2}^{2}-\alpha_{2}^{3}+4 \alpha_{2} \alpha_{3}-\alpha_{3}-4 \alpha_{3}^{2} / \alpha_{2}+\alpha_{4}\right]+O\left(A^{-2}\right) .
\end{aligned}
$$

Equation (14) provides the desired generalization of Rapp's formula. For a known form of input stream distribution function and empirical values for $V_{N}$ and $Z_{N}$, equation (14) gives, on disregarding the $O\left(A^{-2}\right)$ term, a quadratic equation from which $A$ may be obtained as the larger root. In the special case of a Poisson stream, $\alpha_{2}=\alpha_{3}=\alpha_{4}=1$, so that (14) reduces to the linear equation

$$
V_{N}+3 Z_{N}\left(Z_{N}-1\right)=A+O\left(A^{-2}\right) \text {. }
$$

It is clear from (15) that Rapp's formula can be expected to be very accurate indeed in a heavy traffic regime.

An accompanying generalization of (2) may also be derived. Equations (11) and (13) lead at once to

$$
\begin{aligned}
\alpha_{2} N= & \alpha_{2}[A(M+Z) /(M+Z-1)-1-M]+\left(1-\alpha_{2}^{-1}\right) Z\left(Z-\alpha_{2}\right) \\
& +\left(\alpha_{2}-1\right) A^{-1}\left(\alpha_{2}-\alpha_{2}^{2}+\alpha_{3}\right)+O\left(A^{-2}\right) .
\end{aligned}
$$

For a Poisson stream this reduces to equation (2) up to a second order term. 
Equations (14) and (16) provide a generalization of (1) and (2) for general renewal offered traffic under conditions of heavy traffic. Other approximations also exist which are accurate to $O\left(A^{-2}\right)$. Thus we have trivially

$$
\begin{aligned}
V_{N}+ & \alpha_{3}-\alpha_{2}^{2}+\left(1-2 \alpha_{2}+4 \alpha_{3} / \alpha_{2}\right)\left(Z_{N}-\alpha_{2}\right) \\
& =A \alpha_{2}+A^{-1}\left(2 \alpha_{2}^{3}-2 \alpha_{2}^{4}+4 \alpha_{3} \alpha_{2}^{2}-4 \alpha_{3}^{2} / \alpha_{2}-\alpha_{3}+\alpha_{4}\right)+O\left(A^{-2}\right),
\end{aligned}
$$

which reduces to

$$
V_{N}+3\left(Z_{N}-1\right)=A+O\left(A^{-2}\right)
$$

for Poisson offered traffic.

\section{Potter's linear relation}

The following empirical observation has been made by Potter [3]. Let us make explicit the dependence of $M$ and $V$ on the intensity of the offered traffic by expressing them as $M_{N}(A)$ and $V_{N}(A)$. Then, for a variety of forms of renewal input, we have approximately

$$
M_{1+k}(A+k)-M_{1}(A)=s_{A}\left[V_{1+k}(A+k)-V_{1}(A)\right]
$$

where $s_{A}$ does not depend on $k$. This relation is manifested as a collinearity of the points $\left\{\left(M_{1+k}(A+k), V_{1+k}(A+k)\right) ; k>0\right\}$ for each $A$ when $M_{N}$ is graphed against $V_{N}$. Potter's graphs indicate that deviations from linearity are exhibited at low intensities for the offered traffic, suggesting the applicability of our heavy traffic estimates of Section 2 . We shall find that there are, in fact, separate linearities in $M$ and $V$, that is, the distances between consecutive pairs of collinear points are approximately equal. We also obtain an estimate for the value of the coefficient $s_{A}$.

For the evaluation of $M_{1+k}(A+k)$, we note that the change in mean offered traffic from $A$ to $A+k$ can be represented by a change of the holding time parameter from $\mu$ to $\mu_{k}$, where $A+k=-\left[\mu_{k} \phi_{1}\right]^{-1}$, so that by (3) we have $\mu_{k}=\mu /\left(1-k \mu \phi_{1}\right)$. We then obtain $M_{1+k}(A+k)$ from (8) by replacing $N$ by $1+k$ and $\mu$ by $\mu /\left(1-k \mu \phi_{1}\right)$, which results in

$$
\begin{gathered}
M_{1+k}(A+k)=-\left(\mu \phi_{1}\right)^{-1}\left[1+\mu \phi_{1}+\mu^{2} \phi_{21}(1+k) / 2\right. \\
\left.+k(k+1) \mu^{3}\left(\phi_{21}-\phi_{22}\right)+O\left(\mu^{4}\right)\right] .
\end{gathered}
$$

Subtraction of the corresponding result for $k=0$ yields

$$
M_{1+k}(A+k)-M_{1}(A)=k \mu \phi_{21} /\left(-2 \phi_{1}\right)+k(k+1) \mu^{2}\left(\phi_{22}-\phi_{21}\right) \phi_{1}^{-1}+O\left(\mu^{3}\right) \text {, }
$$
displaying a linear dependence on $k$ to first order in $\mu$. If $\phi_{3}$ exists, then $\phi_{22}-\phi_{21}=O(\mu)$ so that

$$
M_{1+k}(A+k)-M_{1}(A)=k A^{-1} \phi_{21} /\left(2 \phi_{1}^{2}\right)+O\left(A^{-3}\right)
$$

and we have linearity to second order. 
Similarly we have

$$
V_{1+k}(A+k)-V_{1}(A)=k\left(2 \phi_{22}-3 \phi_{21} / 2\right) / \phi_{1}^{2}+O\left(A^{-1}\right)
$$

and, if $\phi_{3}$ exists,

$$
\begin{aligned}
& V_{1+k}(A+k)-V_{1}(A) \\
& \quad=k\left[\phi_{21} /\left(2 \phi_{1}^{2}\right)+A^{-1}\left\{2 \alpha_{2}^{2}-\alpha_{2}+(2 / 3)\left(\phi_{31}-2 \phi_{32}\right) / \phi_{1}^{3}\right\}\right]+O\left(A^{-2}\right),
\end{aligned}
$$

where $\phi_{3}$ is defined analogously to $\phi_{2 i}$. Equations (18) and (19) yield

$$
s_{A}=A^{-1}-A^{-2}\left[2 \alpha_{2}-1+(2 / 3)\left(\phi_{31}-2 \phi_{32}\right) /\left(\alpha_{2} \phi_{1}^{3}\right)\right]+O\left(A^{-3}\right)
$$

and, if $\phi_{4}$ exists,

$$
s_{A}=A^{-1}+A^{-2}\left(1-2 \alpha_{2}+4 \alpha_{3} / \alpha_{2}\right)+O\left(A^{-3}\right) .
$$

It can be seen from Table 2 of Potter that the agreement of $s_{A}$ and $A^{-1}$ can be quite good even for conditions of moderate traffic.

\section{References}

[1] J. W. Cohen, "The full availability group of trunks with an arbitrary distribution of the inter-arrival time and the negative exponential holding time distribution", Simon Stevin Wis-en Natuurkundig Tijdschrift 26 (1957), 169-181.

[2] C. E. M. Pearce and R. M. Potter, "Some formulae old and new for overflow traffic in telephony", 8th Int. Teletraffic Congress, Melbourne (1976), 421.1-6 (also with addenda in Aust. Telecom. Res. (1977), 92-97).

[3] R. M. Potter, "The equivalent non-random method and restrictions imposed on renewal overflow systems by the specification of a finite number of overflow traffic moments", 9 th Int. Teletraffic Congress, Torremolinos, Spain (1979), 1-6.

[4] Y. Rapp, "Planning of junction network in a multi-exchange area", Ericsson Technics 20 (1964), 77-130.

[5] L. Takács, Introduction to the theory of queues (Oxford University Press, 1962), Chapters 3, 4.

[6] R. I. Wilkinson, "Theories for toll traffic engineering in the U.S.A.", Bell System Tech. J. 35 (1956), 421-514.

\section{Department of Applied Mathematics}

University of Adelaide

Acielaide

South Australia 5000 